\title{
Pobreza multidimensional intraurbana na região metropolitana de Belém
}

\author{
Danuzia Lima Rodrigues \\ Universidade Federal do Sul e Sudoeste do Pará - Marabá - PA - Brasil \\ ORCID: http://orcid.org/0000-0002-0823-6080 \\ Ricardo Bruno Nascimento dos Santos \\ Universidade Federal do Pará - Belém - PA - Brasil \\ ORCID: http://orcid.org/0000-0001-9459-1582 \\ Izete Pengo Bagolin \\ Pontifícia Universidade Católica do Rio Grande do Sul - Porto Alegre - RS - Brasil \\ ORCID: http://orcid.org/0000-0003-4325-7677 \\ Danilo Araujo Fernandes \\ Universidade Federal do Pará - Belém - PA - Brasil \\ ORCID: http://orcid.org/0000-0003-1326-9626
}

\section{Resumo}

O objetivo deste artigo é analisar as múltiplas dimensões da pobreza intraurbana na Região Metropolitana de Belém, por meio dos microdados do Censo de 2010. Para isso, são utilizados os arcabouços teóricos da Abordagem das Capacitações e da pobreza multidimensional e aplica-se a metodologia de Alkire e Santos (2010). Os resultados evidenciam existência de diferenças entre a pobreza monetária e a pobreza multidimensional e mostram que áreas consideradas menos pobres em termos multidimensionais não são as mesmas quando avaliadas em termos monetários. Percebe-se que as áreas de ponderação, em que praticamente coincidem os indicadores de renda e o multidimensional, não estão na mesma posição na classificação geral dos indicadores sintéticos de avaliação sobre pobreza. Adicionalmente, a composição de privações domiciliares difere entre as áreas de ponderação e a incidência da pobreza multidimensional ainda é elevada, mesmo após os avanços sociais vastamente relatados na literatura nos últimos 15 anos.

Palavras-chave: Pobreza Multidimensional. Metropolitana. Intraurbana.

\section{Abstract}

Multidimensional intra urban poverty in the metropolitan region of Belém

Aiming to analyse the multiple dimensions of intra-urban poverty in the Metropolitan Region of Belém (RMB) we followed the theoretical background of the Capability Approach and multidimensional poverty and used the Alkire and Santos (2010) methodology for the 2010 census micro-data. The results show that income and multidimensional poverty present quite different performances, showing that some areas considered less poor in 
multidimensional terms are not necessarily the least poor when evaluated in terms of income. In addition, the areas of weighting, in which income and multidimensional indicators almost coincide, are not in the same position in the general classification of synthetic indicators of poverty assessment.

Keywords: Multidimensional. Metropolitan. Intra-urban, poverty.

\section{Pobreza multidimensional intraurbana em la región metropolitana de Belém Resumen}

El objetivo de este trabajo es analizar las múltiples dimensiones de la pobreza intraurbana en la Región Metropolitana de Belém. Para eso son utilizados los microdatos del Censo de 2010. Se utilizan los marcos teóricos del Enfoque de Capacidad y pobreza multidimensional y se aplica la metodología Alkire y Santos (2010). Los resultados muestran diferencias entre la pobreza monetaria y la pobreza multidimensional y muestran que las áreas consideradas menos pobres en términos multidimensionales no son las misma cuando se evalúan en términos monetarios. Se observa también que las áreas de ponderación, en las que el ingreso y los indicadores multidimensionales prácticamente coinciden, no están en la misma posición en la clasificación general de los indicadores sintéticos de evaluación de la pobreza. Además, la composición de la privación de los hogares difiere entre las áreas de ponderación y la incidencia de la pobreza multidimensional sigue siendo alta, incluso después de los avances sociales ampliamente reportados en la literatura en los últimos 15 años.

Palabras clave: Pobreza multidimensional. Metropolitana Intraurbano.

\section{Introdução}

O objetivo central deste artigo é analisar as múltiplas dimensões da pobreza intraurbana na Região Metropolitana de Belém (RMB), que é uma região composta por sete municípios, abrange uma área de 3.566 km2 e, em 2010, possuía uma população de, aproximadamente, 2.042,417 habitantes. Belém é o município mais populoso e o que apresenta a maior oferta de serviços e emprego, bem como a concentração de bancos, hospitais, centro de comércio e órgãos públicos, que servem ainda hoje a toda região metropolitana. A interação e o fluxo de pessoas entre Belém e os demais municípios da RMB nesse sentido é intenso, visto que Belém desempenha papel ainda preponderante na economia da região metropolitana.

Com base nos dados disponíveis sobre pobreza monetária e unidimensional (proporção de pobres), é possível perceber que, apesar dos avanços ocorridos no combate à pobreza ao longo dos anos 2000, a RMB ainda apresenta elevada incidência de pobreza, ficando atrás no ranking nacional apenas para as regiões metropolitanas de Recife e Salvador. Além disso, a RMB foi a que apresentou menor taxa de redução na proporção de pobres ao longo do período de 2001 a 2011.

Sabe-se, no entanto, que apesar de sua importância e utilidade, as medidas monetárias e unidimensionais não são suficientes para uma compreensão completa e mais abrangente do fenômeno da pobreza. Em função disso, destaca-se a importância, que a literatura recente tem atribuído aos esforços de construir indicadores multidimensionais de pobreza para subsidiar as decisões e avaliações de política pública. Apesar disso, ainda persiste o desafio de entender a pobreza sob uma perspectiva multidimensional. Essa perspectiva, de olhar para a pobreza 
enquanto um fenômeno multidimensional, complexo e diverso, ganhou força a partir das contribuições de Amartya Sen $(1985,1987,1999)$. A partir destas proposições, a ideia de que a mensuração da pobreza deve ir além de aspectos baseados unicamente na renda foi evoluindo e se consolidando.

De acordo com a Abordagem das Capacitações (AC), a pobreza deve ser compreendida como privação de capacitações básicas e não apenas como insuficiência de renda ou baixo nível de recursos, bens primários ou necessidades básicas (SEN, 1983b; 1984; 1992; 1993; 2000). No entanto, Sen (2000) adverte que o objetivo da $A C$ não é negar os efeitos do baixo nível de renda, e reconhece que baixa renda é uma das causas principais da pobreza. Em Sen (1992: p. 111) ele explica que "ter uma renda inadequada não é uma questão de ter um nível de renda abaixo de uma linha de pobreza fixada externamente, mas de ter uma renda abaixo do que é adequado para gerar os níveis especificados de capacitações para a pessoa em questão". Assim, de acordo com essa perspectiva, o conceito relevante de pobreza precisa contemplar a inadequação da renda para gerar as capacitações minimamente aceitáveis.

Seguindo os argumentos em favor da definição da pobreza como um fenômeno multidimensional, atualmente pode-se assumir que, no que tange à questão conceitual, existe consenso sobre a necessidade de aplicação de critérios de avaliação da sua multidimensionalidade. No entanto, esses avanços conceituais ainda não foram amplamente adotados e incorporados nos processos de identificação e acompanhamento das condições de vida dos pobres, mensuração da incidência e intensidade, bem como na elaboração de políticas de enfrentamento.

Diferentes concepções de pobreza (operacional ou normativa) têm influenciado pesquisadores na busca pela compreensão e identificação de quem são os indivíduos pobres, como eles vivem, qual a intensidade da sua pobreza, dentre outros aspectos (Atkinson 2003, Duclos, Sahn e Younger 2006, Alkire e Foster 2011, Kakwani e Silber, 2008 e 2008b, Alkire e Santos 2010). Dentre as contribuições que buscaram avançar na mensuração da pobreza multidimensional, partindo da AC, duas propostas se destacaram e deram origem, respectivamente, ao Índice de Pobreza Humana (IPH) que foi proposto e utilizado pelas nações unidas até o período em que foi substituído pelo Índice de Pobreza Multidimensional (IPM), proposto por Alkire \& Foster (2011) e que, desde 2014, integra os Relatórios de Desenvolvimento Humano das Nações Unidas.

De acordo com Alkire e Santos (2010), o IPM se apoia na ideia das capacitações de Amartya Sen e se baseia em indicadores que propõem uma estratégia multidimensional mais ampla, capaz de refletir carências básicas, serviços e funcionamentos humanos que são considerados centrais em qualquer avaliação atual sobre a qualidade de vida de uma população ou indivíduos. Com isso, o IPM diferencia-se de outros índices que avaliam a pobreza em seus aspectos multidimensionais, na medida em que ilustra um conjunto combinado mais amplo de carências e privações das famílias. Todavia, o IPM pode, ao mesmo tempo, ser considerado como um índice limitado em sua capacidade de servir como elemento de comparação entre países, por exemplo, pois exige que os mesmos indicadores estejam disponíveis para todos os países incluídos em uma análise comparativa sobre pobreza, além da necessidade de que os dados sejam provenientes da mesma família, obstruindo, portanto, a utilização complementar de outras bases de dados. 
Apesar dessas limitações, desde seu surgimento, o IPM tem sido amplamente testado e adaptado às necessidades e bases de dados de diferentes países, tanto desenvolvidos quanto em desenvolvimento. Por exemplo, foi aplicado para Bósnia and Herzegovina (CHZEHEN e FERRONE, 2017), para a Austrália (CALLANDER et all, 2012). Na América Latina o MPI foi utilizado para calcular pobreza multidimensional na Nicarágua (MONTOYA e Teixeira, 2017) e na Colômbia (ÂNGULO, et all, 2016). Na Ásia, foi utilizado na Malálisa (SOLAYMANI e KARI, 2014), no Paquistão (SABBOR, et, al, 2015; KHAN, et all, 2014), na China (QI e WY, 2015) e Índia (ALKIRE e SETH, 2015). Também foi utilizado no continente Africano, em Angola (PINILLA-RONCANCIO e SILVA, 2018), na África do Sul (MUSHONGERA et al, 2017), na Uganda (LEVINE et all, 2014).

Especificamente para o Brasil, os trabalhos de Vaz e Jannuzzi (2014), usando dados da PNAD, combinaram os critérios do IPM global para estimar a pobreza multidimensional entre 2001 e 2013. Jannuzzi e Sousa (2016), calcularam o IPM Cepal-Sagi para captar os efeitos da conjuntura econômica e resultados das políticas sociais entre 1992 e 2014, entre os setores urbano e rural. Já o trabalho de Serra (2017), construiu um IPM para o Brasil visando analisar as mudanças na pobreza multidimensional entre 2000 a 2010, comparando as áreas rurais e urbanas.

No entanto, para a Região Norte, e para o estado do Pará, em específico, poucos estudos utilizando indicadores multidimensionais sintéticos de pobreza foram realizados nos últimos anos. No que diz respeito à situação específica da RMB, os trabalhos mais significativos têm concentrado esforços na compreensão de indicadores de emprego, saúde e educação, de maneira separada, ou a partir de estudos de caso em determinadas regiões específicas; sem que para isso se tenha avançado de maneira significativa na construção e aplicação de indicadores sintéticos amplos para efeito de comparação entre regiões intraurbanas e entre municípios da região metropolitana de Belém.

De uma maneira geral, de acordo com Santos (2015), a RMB é uma das regiões metropolitanas do Brasil que apresenta as maiores taxas de desemprego e fragilidade ocupacional do país. Apesar disso, entre 2000 e 2010, ela vinha apresentando queda em seus níveis de informalidade e também no número de trabalhadores desocupados. Esses dados parecem corroborar as análises que dão conta de uma melhoria nos níveis médios de renda e emprego da população da RMB entre os anos de 2000 e 2010. No entanto, quando avaliamos indicadores relacionados à saúde, educação e saneamento, por exemplo, os resultados parecem bem menos satisfatórios.

No que diz respeito à educação, Santos (2015) avalia que apesar de alguma melhoria nos indicadores em 2010, a RMB continuava apresentando resultados bastante ruins quando comparados à outras regiões metropolitanas do país. Da mesma forma, tanto indicadores de saúde em geral, como os de saneamento de maneira específica têm sido considerados um dos piores do país.

De acordo com o Atlas do Desenvolvimento Humano (2013), por exemplo, a RMB apresentava um IDHM de 0,729 em 2010. No entanto, quando são analisados os IDHMs dos municípios que compõem a RMB, percebe-se realizações humanas bastante distintas, com o município de Belém alcançando um IDHM de 0,746, enquanto que o município de Santa Bárbara alcança um IDHM de apenas 0,505. Além das diferenças de realizações no IDHM médio entre os municípios da RMB, é 
importante destacar que as realizações em cada uma das dimensões que compõem o índice, diferem tanto entre os municípios quanto dentro de cada município. Neste sentido, destaca-se que apesar do IDHM representar um avanço importante em termos de análise de bem-estar, contemplando a multidimensionalidade das realizações, ele não é ainda uma medida suficientemente adequada para se pensar e avaliar a pobreza em seus aspectos multidimensionais.

Com base nesses princípios metodológicos, o presente trabalho faz uso do IPM como medida sintética alternativa, buscando contribuir para uma compreensão mais completa da diversidade da pobreza urbana da RMB, nos seus aspectos intra e intermunicipais. Para isso, são utilizados os dados de 2010 das áreas de ponderação do Censo do IBGE. A utilização das áreas de ponderação permitirá uma visão mais ampla e, inclusive, espacializada da pobreza multidimensional na região objeto de estudo. Esse tipo de abordagem é importante, pois fornece informações mais detalhadas que permitem diagnósticos mais amplos da pobreza, o que pode contribuir significativamente no sentido de se elaborar políticas públicas mais efetivamente direcionadas a uma determinada localidade.

Além desta introdução, o artigo apresenta a metodologia do IPM e as adaptações necessárias que foram realizadas para o estudo sobre os níveis de pobreza na RMB; a apresentação e análise dos resultados alcançados e as considerações finais.

\section{Dados e metodologia}

De acordo com Alkire e Santos (2010), as possibilidades de escolha de dimensões capazes de refletir a pobreza são bastante amplas. Várias dimensões, como saúde, educação, padrão de vida, empoderamento, condições de emprego e trabalho, meio ambiente, proteção contra a violência, relacionamentos sociais e culturais, entre outras, são em geral levadas em consideração quando se debate os possíveis aspectos a serem considerados em uma avaliação sobre a pobreza multidimensional.

No contexto deste estudo, o que se propõe é adotar como ponto de partida a metodologia desenvolvida por Alkire e Santos (2010), que foi utilizada em um primeiro exercício para se estimar a pobreza multidimensional para 100 países em desenvolvimento. Essa metodologia, chamada de IPM, apresenta a mesma estrutura matemática baseada no método proposto originalmente por Alkire e Foster (2011) e que foi utilizada pelo PNUD no Relatório de Desenvolvimento Humano de 2013 (PNUD, 2013).

O método desenvolvido por Alkire e Foster ( 2011), tendo como escopo a abordagem das capacitações de Amartya Sen, possibilita o cálculo tanto da incidência quanto da intensidade da pobreza, capturando uma distribuição conjunta de privações. Segundo os autores, do ponto de vista técnico e funcional, esse método é relevante, visto que a família de medidas AF são axiomáticas e satisfazem várias propriedades desejáveis. Além disso, é funcional, uma vez que utiliza a contagem intuitiva e leva em consideração a maneira como se distribuem as privações para identificar os pobres em termos multidimensionais.

Seguindo Sen (1976), que argumenta que a mensuração da pobreza exige o enfrentamento de dois problemas, que são a identificação de quem é pobre e a 
construção do índice de pobreza, a metodologia AF - particularmente a construção da medida Mo - é sistematizada em duas etapas: identificação e agregação.

$\mathrm{Na}$ etapa da identificação, o primeiro passo é definir a unidade de análise, se indivíduo, família, grupo/subgrupo etc. O segundo e terceiro passos seriam as definições das dimensões e de seus respectivos conjuntos de indicadores que serão considerados na medida multidimensional (os dados referentes à unidade de análise i e dimensões j podem ser disponibilizados em uma matriz Yij). O quarto passo seria determinar os pontos de corte, ou linhas de pobreza por indicador, para verificar se cada pessoa é privada ou não em cada indicador (ALKIRE E FOSTER, 2011)

A metodologia geral de identificação supracitada usa o método de duplo corte proposto pelos autores para a identificação dos pobres. No primeiro corte, busca-se definir se a unidade de análise enfrenta privação, ou não, em determinado indicador. Posteriormente, constrói-se uma matriz de vetor linha z com as linhas de corte de pobreza estabelecidas. Convém antecipar que o segundo corte é realizado posteriormente sobre a soma das privações de cada unidade de análise.

O próximo passo (quinto) é fazer a ponderação das dimensões e definir uma matriz de privações que será construída a partir da comparação entre as matrizes Yij e $z$, em que as unidades de análise consideradas privadas receberão valores 1 e as não privadas receberão valor zero. Ou seja, a matriz resultante $\mathrm{g}^{\circ}$ será uma matriz binária formada por zeros e uns.

Vale ressaltar que, de acordo com Alkire e Santos (2010), há três maneiras de se aplicar os pesos em análises sobre pobreza multidimensional: a) entre as dimensões (o peso relativo da saúde e educação), b) dentro das dimensões (se for utilizado mais de um indicador) e c) entre os indivíduos na distribuição (para priorizar, por exemplo, os mais desfavorecidos). O indicador que será aqui utilizado (IPM) atribui os pesos de maneira igual em cada dimensão, e da mesma forma cada indicador dentro da dimensão. ${ }^{1}$

O passo seis é criar a soma ponderada das privações para cada unidade de análise, e a partir da matriz $\mathrm{g}^{0}$ construir um vetor coluna $\mathrm{c}$ que representa a soma ponderada de privações sofridas por cada unidade de análise i. Só assim define-se o segundo corte $k$, que é a proporção de privações ponderadas que uma pessoa precisa experimentar para ser considerada multidimensionalmente pobre, ou seja, 0 número mínimo de indicadores que a unidade deve ser privada para ser considerada pobre. Posteriormente, aplica-se esse número na matriz $c$ e, com base nela, novamente se constrói outra matriz binária censurada $\mathrm{g}^{\circ}(\mathrm{k})$, colocando 1 para a unidade que está acima ou igual ao ponto de corte $k$ (pobres) e zero para quem está abaixo (não pobres).

Em termos formais, isso nada mais é do que uma função de identificação $\rho_{k}$ : $R_{+}^{d} \times R_{++}^{d} \rightarrow[0,1]$, que mapeia as realizações da unidade de análise i no vetor Yi $\in R_{+}^{d}$

\footnotetext{
${ }^{1}$ Com relação à atribuição de pesos aos indicadores e dimensões, existe uma discussão se esses pesos homogêneos são melhores ou não. Segundo Alkire e Santos (2010), a decisão de determinar o mesmo peso para as dimensões que compõem o IPM surgiu após várias discussões, testes de robustez, opinião de analistas e também de análises participativas. Vale destacar que apesar de o IPM permitir a utilização de diferentes pesos para cada dimensão ou indicador, os autores da metodologia atribuem pesos iguais em cada dimensão e igualmente para cada indicador selecionado dentro das dimensões.
} 
e o corte vetorial $z$ em $R_{++}^{d}$ para cada variável indicada. Sendo que $\rho_{k}$ assume valor 1 quando $\mathrm{ci} \geq \mathrm{k}$ e $\rho_{k}(\mathrm{Yi}, \mathrm{z})=0$ quando $\mathrm{ci}<\mathrm{k}$. Dessa forma, o cálculo do índice dará ênfase apenas às unidades que forem classificadas como pobres (1), censurando as demais.

Para a etapa de agregação, a metodologia de Alkire e Foster (2011) também segue as ideias de Sen (1976). Neste trabalho, após todos os passos de identificação elaborados, se calcula o headcount, ou seja, a proporção de pessoas que foram identificadas como multidimensionalmente pobres na população $(H)^{2}$ :

$$
H=\frac{q}{n}
$$

Onde:

q é o número de pessoas multidimensionalmente pobres.

n é a população total.

A intensidade da pobreza média compartilhada entre pobres implica em somar os escores de privação dos pobres e dividi-los pelo número total de pessoas pobres:

$$
A=\frac{\sum_{1}^{q} c}{q}
$$

Onde:

c é a contagem de carências experimentadas pelos pobres.

O passo seguinte é calcular a medida Mo, que resume a informação sobre a incidência de pobreza e sua intensidade. Daí o nome de índice de incidência ajustado. O Adjusted Headcount Ratio (Mo), reflete a proporção de privações ponderadas que os pobres experimentam em relação ao número total de privações que a população poderia experimentar se todas as pessoas fossem pobres e fossem privada em todas as dimensões consideradas na análise.

$$
\mathrm{IPM}=\mathrm{A} \times \mathrm{H}
$$

A contribuição de cada dimensão j à pobreza multidimensional é:

$$
\text { Contrib }_{j}=\frac{\left(\sum_{1}^{q} c_{j}\right) / n}{I P M}
$$

O indicador construído para análise da pobreza multidimensional da RMB baseia-se em Alkire e Santos (2010), mas faz as devidas adaptações mediante as especificidades do objeto de estudo e a disponibilidade de dados. Todas as dimensões que constituem o índice, no entanto, foram analisadas e identificadas como importantes na composição do bem-estar das pessoas, estando em conformidade direta ou indiretamente com os Objetivos de Desenvolvimento do Milênio (ODM), por isso permaneceram como proposta inicial nesta análise. Além disso, levou-se em consideração que os temas são amplamente utilizados na literatura, o que facilita, portanto, a interpretação e análise do IPM. O índice inclui três dimensões: saúde, educação e padrão de vida. Dito isso, a unidade de análise

\footnotetext{
${ }^{2}$ Alkire \& Santos (2009) salientam que Headcount não é sensitivo com o número de privações que o pobre enfrenta.
} 
considerada será o agregado familiar, sendo possível também identificar o número de indivíduos que vivem em agregados familiares multidimensionalmente pobres.

Os indicadores utilizados no IPM contemplam três dimensões e englobam oito indicadores, que são: Ensino Fundamental incompleto (+18 anos); crianças em idade escolar que não frequentam a escola (4 a 14 anos); água potável; total de filhos nascidos mortos (natimorto); eletricidade; esgotamento sanitário; destino do lixo; se tem carro, freezer, telefone e televisão.

O IPM reflete o número de privações conjuntas que o indivíduo sofre. Dessa forma, indivíduos que apresentam não necessariamente todas, mas, sim, um conjunto de privações básicas, devem ser considerados pobres. Empiricamente, cada indivíduo recebe a contagem de carências que sofre em cada um dos indicadores. Sendo que a contagem máxima de carências é de $100 \%$, cada dimensão recebe a mesma ponderação (1/3). Dessa maneira, a contagem máxima em cada dimensão será de $33,33 \%$, isso porque no IPM é composto por três dimensões. Cada indicador será ponderado, dividindo a ponderação (1/3) pelo número de indicadores.

Para identificar os indivíduos multidimensionalmente pobres, deve-se somar as carências de cada um para obter, posteriormente, a carência total correspondente de cada indivíduo, C. Com o intuito de diferenciar pobres e não pobres, admite-se como ponto de corte da pobreza $(k)^{3}$ o valor de $33,33 \%$ correspondente à terceira parte dos indicadores ponderados. Ou seja, o corte determinado para a identificação dos indivíduos pobres é de que haja privação em, pelo menos, 33,33\% dos indicadores. Assim, serão assumidos os seguintes critérios:

i. Se $C$ for $\geq 33,3 \%$, os indivíduos são considerados pobres multidimensionalmente.

ii. Se $C$ for $\geq 20 \%$ e $<33,3 \%$, os indivíduos são vulneráveis ou estão em risco de cair na pobreza multidimensional

iii. Se $C \geq 50 \%$, os indivíduos sofrem de pobreza multidimensional extrema.

Assim, conforme a contagem de carências dos indivíduos, eles vão sendo classificados quanto a sua condição de pobreza multidimensional. O valor do IPM é a média das contagens de carência C (superiores a 33,3\%) da população. A Tabela 1, a seguir, sintetiza o IPM.

\footnotetext{
${ }^{3}$ A variável k é o ponto de corte da pobreza e reflete a soma de indicadores ponderados no qual o indivíduo deve ser privado para ser considerado multidimensionalmente pobre. Vale ressaltar que esse parâmetro é flexível, portanto pode ser analisado em seus vários valores de acordo com o número de dimensões que se quer considerar.
} 
Tabela 1 - Dimensões, indicadores, pontos de corte e ponderações do IPM da RMB

\begin{tabular}{|c|c|c|c|}
\hline Dimensão & Indicadores & Ponto de corte & Ponderação \\
\hline \multirow{2}{*}{ Educação } & $\begin{array}{l}\text { Ensino Fundamental } \\
\text { incompleto }\end{array}$ & $\begin{array}{l}\text { Existência de indivíduos acima de } 18 \\
\text { anos que não estudaram ou não } \\
\text { concluíram o Ensino Fundamental }\end{array}$ & $1 / 3 \div 2=16,7 \%$ \\
\hline & $\begin{array}{l}\text { Crianças em idade } \\
\text { escolar que não } \\
\text { frequentam a escola }\end{array}$ & $\begin{array}{l}\text { Existência de crianças de } 4 \text { a } 14 \text { anos } \\
\text { que não estão sendo expostas a um } \\
\text { ambiente de aprendizagem }\end{array}$ & $1 / 3 \div 2=16,7 \%$ \\
\hline \multirow{2}{*}{ Saúde } & Água potável & $\begin{array}{l}\text { Inexistência de abastecimento de } \\
\text { água procedente da rede geral de } \\
\text { distribuição }\end{array}$ & $1 / 3 \div 2=16,7 \%$ \\
\hline & $\begin{array}{l}\text { Total de filhos nascidos } \\
\text { mortos (natimorto) }\end{array}$ & $\begin{array}{l}\text { Se no agregado familiar há pelo } \\
\text { menos um natimorto }\end{array}$ & $1 / 3 \div 2=16,7 \%$ \\
\hline \multirow{4}{*}{$\begin{array}{l}\text { Condições de } \\
\text { vida }\end{array}$} & Eletricidade & $\begin{array}{l}\text { Inexistência de energia elétrica no } \\
\text { domicílio }\end{array}$ & $1 / 3 \div 4=8,33 \%$ \\
\hline & Esgotamento sanitário & $\begin{array}{l}\text { Domicílios desprovidos de } \\
\text { esgotamento sanitário via rede geral } \\
\text { e fossa séptica }\end{array}$ & $1 / 3 \div 4=8,33 \%$ \\
\hline & Destino do lixo & $\begin{array}{l}\text { Inexistência de coleta de lixo por } \\
\text { serviço de limpeza ou caçamba de } \\
\text { serviço de limpeza }\end{array}$ & $1 / 3 \div 4=8,33 \%$ \\
\hline & $\begin{array}{l}\text { Se tem carro, freezer, } \\
\text { telefone e televisão }\end{array}$ & $\begin{array}{l}\text { Inexistência de, pelo menos, algum } \\
\text { desses bens de consumo no } \\
\text { domicílio }\end{array}$ & $1 / 3 \div 4=8,33 \%$ \\
\hline
\end{tabular}

Resultados

Contagens de carências do indivíduo, C (soma de cada carência multiplicada por sua ponderação)

É considerado pobre o indivíduo (C > 33,3\%)

Fonte: Elaboração própria com base nos censos 2000 e 2010.

As variáveis utilizadas têm origem nos microdados do Censo de 2010 referentes as 73 áreas de ponderação (Figura 1), divididas entre os diferentes municípios da Região Metropolitana de Belém (Belém, Ananindeua, Marituba, Benevides, Santa Izabel, Santa Bárbara e Castanhal). A análise concentra-se, no entanto, nas 20 áreas de ponderação (APs) selecionadas, sendo dividida em duas partes: na primeira metade, classificam-se as 10 melhores no ranking; na outra metade são consideradas as 10 piores classificações (das 73 áreas que compõem a $\mathrm{RMB}$ ). O número total de domicílios na RMB é de 601.635 para 2.042.417 pessoas. Os dados revelam que quase metade dos habitantes são pobres de acordo com o IPM, o que corresponde a aproximadamente $46,93 \%$ do total. 
A Figura 1, a seguir, mostra a subdivisão dos municípios da RMB por áreas de ponderação (AP). Como frisado anteriormente, o IPM se diferencia das análises tradicionais, na medida em que considera como multidimensionalmente pobre os indivíduos que sofrem privações em determinada combinação de indicadores. Logo, o indivíduo que for considerado como sofrendo privações em algum indicador específico só será considerado pobre se atingir a contagem de carências consideradas suficientes por um conjunto de indicadores agregados.

Figura 1 - Municípios da RMB divididos conforme suas áreas de ponderação

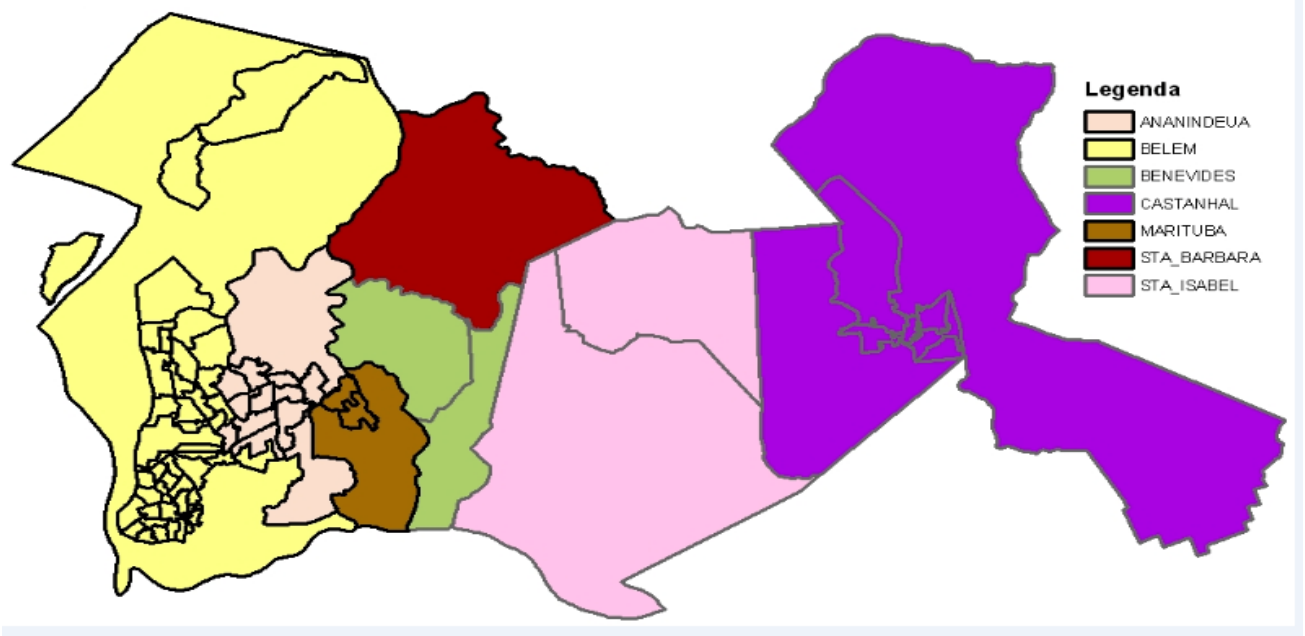

Fonte: Elaborado pelos autores.

Na seção seguinte, são apresentados e discutidos os principais resultados, tendo como referências as regiões de ponderação da Figura 1.

\section{Resultados e discussão}

Na Tabela 2, a seguir, são apresentados os resultados para as 20 APs selecionadas de acordo com o ordenamento do IPM. A ênfase inicial será nos subíndices que compõem o IPM, principalmente a incidência $(H)$ que possibilita a avaliação da proporção da pobreza entre as diferentes APs. Além disso, são apresentados os índices de pobreza de renda, assim como seus rankings, possibilitando a comparação entre este indicador e a proporção de pobres multidimensionais para o ano de 2010. Dentre as 73 APs verifica-se que na capital paraense há pelo menos 10 APs com posições acima da $60^{a}$ no ranking para toda a $\mathrm{RMB}$, o que permite dizer que nesse município há APs em situação bastante delicada no quesito pobreza multidimensional.

Apesar disso, Belém mostra-se bastante diversificada, pois há áreas muito bem classificadas para o padrão da RMB, como a AP Nazaré, que apresenta a melhor posição nesse município obtendo a $5^{\text {a }}$ colocação, com um IPM de 0.0905 (ou 9,05\%), que é um epítome dos $23 \%$ de pobres multidimensionais dessa área, isto é, a proporção de pobres ajustada pela intensidade da pobreza. Ananindeua, que é o segundo município mais populoso da região, apresenta a AP melhor classificada no ranking; no Centro Júlia Seffer, que apresenta um IPM de 0,0692, das 34.844 pessoas dos agregados familiares que ali residem apenas $17 \%$ são classificadas como 
pobres multidimensionalmente. A área que foi apontada como a mais pobre foi a AP de Santa Bárbara, em que, aproximadamente, 77\% dos 17.139 moradores vivem em situação de privação domiciliar múltipla, e as famílias são pobres em média em 43\% dos indicadores. Isso significa que, na média, as famílias são apresentam privações em mais de uma dimensão, mas também em vários indicadores, visto que a privação de cada dimensão é em torno de 33,33\%.

Com relação à proporção de famílias que vivem com menos de $\mathrm{R} \$ 140,56$ por mês (pobres) ou com menos de R\$ 70,28/mês (extremamente pobres) em termos de rendimento comparado com a proporção de pobres multidimensionais mensurados pelo IPM, o que se observou é que em apenas uma área de ponderação localizada em Ananindeua (Aurá e Águas Brancas) a pobreza de renda é maior em termos percentuais que a pobreza multidimensional, em torno de $27 \%$ e $24 \%$, respectivamente. Dentro dessa AP, a localidade do Aurá é conhecida por possuir uma ampla área com um lixão a céu aberto, onde grande parte das famílias, incluindo crianças, trabalha como catadores para manter seu sustento. Há situações em algumas APs, tanto em Belém quanto em Ananindeua, que essas proporções são bastante extremas. Como exemplo, pode-se citar a AP Cidade Nova em Ananindeua, onde a proporção de famílias que sofrem de múltiplas privações é de $45 \%$, enquanto que os pobres em termos de rendimento somam apenas $6 \%$. Nessa AP, a renda per capita é de $R \$ 1.587,98$, e o nível de concentração de renda é de 0,45 . No município de Belém, pode-se mencionar a AP do Umarizal, em que o percentual de famílias que vivem com $R \$ 140,56 /$ mês é de apenas $3 \%$, ao passo que 0 percentual de incidência da pobreza multidimensional é de 31\%. A renda média nessa AP é altamente concentrada, pois a renda per capita e o índice de Gini são, respectivamente, de $\mathrm{R} \$ 4 \cdot 371,41$, e 0,51. A AP do Umarizal é bastante díspar, nela é possível identificar um espaço mais nobre, onde residem famílias de alto poder aquisitivo, ladeado por áreas insalubres e extremamente carentes, isso pode explicar a renda per capita alta e, consequentemente, sua elevada concentração.

Assim, de acordo com a Tabela 2, a seguir, a AP que ocupa o $1^{\circ}$ lugar na classificação, isto é, a menos pobre e extremamente pobre de renda das 73 que compõem a RMB, fica no município de Belém (Umarizal), já em termos do IPM, sua colocação no ranking é a $12^{\mathrm{a}}$. Resultado que sugere possíveis disparidades entre as análises que enfatizam a noção de pobreza apenas via uma dimensão (renda) e análises que compreendem a pobreza como uma soma de privações sociais. Os dados informam que a AP mais pobre em termos uni e multidimensional se localiza em Santa Bárbara, ocupando a última posição no ranking $\left(73^{\mathrm{a}}\right)$, onde $77 \%$ de sua população sofre de privações domiciliares múltiplas e $40 \%$ vivem com apenas $\mathrm{R} \$$ 140,56 por mês. 
Tabela 2 - Rankings do IPM e comparações de renda para as 10 melhores e as 10 piores classificações (2010)

\begin{tabular}{|c|c|c|c|c|c|c|c|c|}
\hline \multirow{3}{*}{ AP } & \multicolumn{4}{|c|}{ Pobreza Multidimensional Aguda } & \multicolumn{4}{|c|}{ Pobreza de Renda } \\
\hline & \multirow{2}{*}{$\begin{array}{c}\text { IPM } \\
\text { (Valor) }\end{array}$} & \multirow{2}{*}{$\begin{array}{c}\text { IPM } \\
\text { (Ranking) }\end{array}$} & \multirow{2}{*}{$\mathrm{H}_{1}$ * } & \multirow{2}{*}{$\mathrm{A} 1 * *$} & \multicolumn{2}{|c|}{$\begin{array}{l}\text { Proporção de } \\
\text { pobres }\end{array}$} & \multicolumn{2}{|c|}{$\begin{array}{l}\text { Proporção de } \\
\text { indigentes }\end{array}$} \\
\hline & & & & & Valor & Ranking & Valor & $\begin{array}{c}\text { Rankin } \\
\text { g }\end{array}$ \\
\hline $\begin{array}{l}\text { Centro Júlia } \\
\text { Seffer } \\
\text { Heliolandia }\end{array}$ & 0.0692 & 1 & 0.1795 & 0.3856 & 0.1816 & 39 & 0.12 & 61 \\
\hline $\begin{array}{l}\text { e Geraldo } \\
\text { Palmeira }\end{array}$ & 0.0738 & 2 & 0.1965 & 0.3753 & 0.1874 & 41 & 0.0736 & 36 \\
\hline $\begin{array}{l}\text { Coqueiro } \\
\text { Providência } \\
\text { Jiboia }\end{array}$ & 0.0887 & 3 & 0.2287 & 0.3881 & 0.0808 & 8 & 0.02 & 1 \\
\hline $\begin{array}{l}\text { Branca e } 40 \\
\text { Horas }\end{array}$ & 0.0892 & 4 & 0.2160 & 0.4127 & 0.203 & 48 & 0.0699 & 34 \\
\hline Nazaré & 0.0905 & 5 & 0.2334 & 0.3877 & 0.041 & 2 & 0.0364 & 5 \\
\hline $\begin{array}{l}\text { Coqueiro } \\
\text { Norte }\end{array}$ & 0.0906 & 6 & 0.2328 & 0.3894 & 0.0837 & 9 & 0.0468 & 14 \\
\hline $\begin{array}{l}\text { Aurá e } \\
\text { Águas } \\
\text { Brancas }\end{array}$ & 0.0949 & 7 & 0.2431 & 0.3905 & 0.2762 & 63 & 0.1424 & 67 \\
\hline Icuí & 0.1035 & 8 & 0.2543 & 0.4072 & 0.205 & 49 & 0.0966 & 54 \\
\hline São Brás & 0.1050 & 9 & 0.2736 & 0.3839 & 0.043 & 3 & 0.0322 & 4 \\
\hline $\begin{array}{l}\text { Centro } \\
\text { Histórico }\end{array}$ & 0.1184 & 10 & 0.3045 & 0.3890 & 0.0855 & 10 & 0.0722 & 35 \\
\hline Condor & 0.2530 & 64 & 0.6674 & 0.3791 & 0.1779 & 37 & 0.0947 & 53 \\
\hline Guamá-01 & 0.2718 & 65 & 0.6711 & 0.4051 & 0.1472 & 28 & 0.0454 & 12 \\
\hline $\begin{array}{l}\text { Distrito } 05 \\
\text { de } \\
\text { Benevides }\end{array}$ & 0.2726 & 66 & 0.6360 & 0.4286 & 0.2026 & 47 & 0.0929 & 52 \\
\hline Jurunas-02 & 0.2924 & 67 & 0.7524 & 0.3886 & 0.165 & 31 & 0.0562 & 19 \\
\hline Terra Firme & 0.2991 & 68 & 0.7209 & 0.4149 & 0.21 & 51 & 0.085 & 46 \\
\hline Guamá-03 & 0.3014 & 69 & 0.7370 & 0.4090 & 0.2242 & 54 & 0.0914 & 49 \\
\hline Barreiro & 0.3028 & 70 & 0.7796 & 0.3884 & 0.1978 & 44 & 0.0693 & 30 \\
\hline $\begin{array}{l}\text { Santa Isabel } \\
\text { Área } 001\end{array}$ & 0.3098 & 71 & 0.7267 & 0.4263 & 0.3226 & 70 & 0.1043 & 56 \\
\hline $\begin{array}{l}\text { Castanhal } \\
\text { Área } 003\end{array}$ & 0.3212 & 72 & 0.7568 & 0.4244 & 0.4006 & 72 & 0.1983 & 73 \\
\hline $\begin{array}{l}\text { Santa } \\
\text { Bárbara } 001\end{array}$ & 0.3413 & 73 & 0.7775 & 0.4389 & 0.4047 & 73 & 0.1621 & 70 \\
\hline
\end{tabular}

Fonte: Elaboração própria com base nos microdados do Censo 2010

* Proporção de pobres multidimensionais; ** Intensidade de privações

Outro aspecto no qual o IPM se destaca é a possibilidade de se verificar as contribuições relativas de cada dimensão ou de cada indicador dentro da dimensão para a pobreza multidimensional, a partir de sua decomposição. Isso permite aos formuladores de políticas públicas um melhor entendimento de como se configura a pobreza em cada região e a proposição de formas mais efetivas de combatê-la. 
A Tabela 3, a seguir, fornece as contribuições dimensionais de cada AP à sua pobreza total. A soma do contributo de cada indicador de privação dá a contribuição de cada dimensão, o que proporciona uma visão geral do perfil das APs consideradas pobres. Dessa forma, as dimensões que mais contribuíram para a pobreza multidimensional na RMB em 2010 foram as dimensões saúde e educação. Entre elas, a dimensão que se sobressai como a que mais influenciou nas medidas do IPM foi a dimensão saúde, visto que em 42 das 73 APs da RMB, essa dimensão foi a que mais contribuiu para a pobreza multidimensional aguda. Isso indica que a RMB ainda sofre com sérios problemas em relação ao consumo de água potável e com o acompanhamento pré-natal de futuras mães, visto que o indicador natimorto também compõem essa dimensão.

Nas demais APs, a dimensão que mais influenciou no índice foi a educação, com destaque para Castanhal, pois nesse município a dimensão educação predominou em todas as áreas. Isso representa um aspecto preocupante, pois considerando o fato de que crianças em idade escolar estão fora da escola e que adultos possuem apenas o Ensino Fundamental completo (ou nem isso), pode-se considerar que esses indicadores apontam para um comprometimento estrutural em relação à capacidade futura dessas famílias que habitam na RMB, no sentido de suas condições de alcançar melhores trabalhos, maiores salários e mais dignidade em termos de condições de vida, visto por seus aspectos múltiplos.

Os dados revelam que a AP que apresentou o maior percentual de contribuição na dimensão saúde, em torno de 51\%, foi a do Umarizal, em Belém, mostrando que parte das famílias que residem nessa área sofre de acentuada privação nos indicadores de saúde. Isso pode ter conexão com o fato de que essa AP é ainda composta em grande parte por palafitas, o que influencia substancialmente no indicador água potável, bem como indiretamente no indicador de número de natimortos. Já a maior contribuição percentual da dimensão educação é identificada na AP denominada Heliolândia e Geraldo Palmeira, em Ananindeua, com uma contribuição de $52 \%$ na composição do índice. A mais alta contribuição da dimensão padrão de vida foi observada na AP denominada de Área Rural em Belém (33\%), sugerindo que as famílias dessa área sofrem forte privação de alguma combinação de indicadores que compõem a referida dimensão. O que condiz com o fato de que, por ser uma área rural, pode haver uma maior probabilidade de que indicadores como eletricidade, destino do lixo e saneamento tenham influenciado, consideravelmente, no percentual de contribuição. 
Tabela 5- Contribuição de privações para as 10 melhores e as 10 piores classificações (2010)

\begin{tabular}{|c|c|c|c|c|c|c|}
\hline \multirow[b]{2}{*}{ AP } & \multirow[b]{2}{*}{$\begin{array}{c}\text { IPM } \\
\text { (Valor) }\end{array}$} & \multirow[b]{2}{*}{$\begin{array}{c}\text { IPM } \\
\text { (Ranking) }\end{array}$} & \multicolumn{3}{|c|}{$\begin{array}{l}\text { Percentual de contribuição das } \\
\text { privações em: }\end{array}$} & \multirow[b]{2}{*}{ Carpon } \\
\hline & & & Educação & Saúde & $\begin{array}{l}\text { Padrão de } \\
\text { vida }\end{array}$ & \\
\hline Centro Júlia Seffer & 0.0692 & 1 & 0.4362 & 0.3718 & 0.1919 & 0.1800 \\
\hline $\begin{array}{l}\text { Heliolandia e Geraldo } \\
\text { Palmeira }\end{array}$ & 0.0738 & 2 & 0.5201 & 0.3496 & 0.1303 & 0.1754 \\
\hline Coqueiro Providência & 0.0887 & 3 & 0.3909 & 0.4447 & 0.1644 & 0.2022 \\
\hline $\begin{array}{l}\text { Jiboia Branca e } 40 \\
\text { Horas }\end{array}$ & 0.0892 & 4 & 0.4120 & 0.3661 & 0.2218 & 0.2015 \\
\hline Nazaré & 0.0905 & 5 & 0.3687 & 0.5102 & 0.1211 & 0.2100 \\
\hline Coqueiro Norte & 0.0906 & 6 & 0.4069 & 0.4355 & 0.1576 & 0.1759 \\
\hline Aurá e Águas Brancas & 0.0949 & 7 & 0.4752 & 0.3045 & 0.2202 & 0.2097 \\
\hline Icuí & 0.1035 & 8 & 0.4642 & 0.3072 & 0.2287 & 0.2299 \\
\hline São Brás & 0.1050 & 9 & 0.3784 & 0.4933 & 0.1283 & 0.2304 \\
\hline Centro Histórico & 0.1184 & 10 & 0.3965 & 0.4997 & 0.1038 & 0.2350 \\
\hline Condor & 0.2530 & 64 & 0.4415 & 0.4977 & 0.0608 & 0.3006 \\
\hline Guamá-01 & 0.2718 & 65 & 0.4096 & 0.4892 & 0.1012 & 0.3138 \\
\hline Distrito 05 de Benevides & 0.2726 & 66 & 0.3778 & 0.4065 & 0.2157 & 0.3343 \\
\hline Jurunas-02 & 0.2924 & 67 & 0.4534 & 0.4801 & 0.0664 & 0.3177 \\
\hline Terra Firme & 0.2991 & 68 & 0.4071 & 0.4590 & 0.1339 & 0.3379 \\
\hline Guamá-03 & 0.3014 & 69 & 0.4326 & 0.4454 & 0.1220 & 0.3380 \\
\hline Barreiro & 0.3028 & 70 & 0.4496 & 0.4969 & 0.0535 & 0.3292 \\
\hline Santa Isabel Área 001 & 0.3098 & 71 & 0.3906 & 0.3270 & 0.2824 & 0.3554 \\
\hline Castanhal Área 003 & 0.3212 & 72 & 0.4501 & 0.3840 & 0.1659 & 0.3543 \\
\hline Santa Bárbara 001 & 0.3413 & 73 & 0.3697 & 0.3486 & 0.2816 & 0.3813 \\
\hline
\end{tabular}

Fonte: Elaboração própria com base nos microdados do Censo 2010.

O IPM permite classificar as APs quanto a sua condição de pobreza, baseando-se nos parâmetros estabelecidos na metodologia. No que concerne a esta avaliação (carpon), foram detectadas apenas sete áreas de ponderação onde os indivíduos que compõem a família foram considerados não pobres, de acordo com o que foi estipulado pelo IPM. Dentre essas áreas de ponderação, pode-se citar como exemplo as áreas Centro Júlia Seffer, localizada no município de Ananindeua, que foi inicialmente fundada por funcionários públicos possuidores de certo poder aquisitivo e, portanto, a área foi dotada de uma certa infraestrutura (como seu próprio abastecimento e distribuição de água), e a de Nazaré, em Belém (cuja área é considerada nobre, por isso recebe uma atenção especial por parte do poder público), com variações percentuais de $17 \%$ e $21 \%$, respectivamente.

Foram encontradas 60 áreas em risco, ou seja, áreas que apesar de não serem consideradas pobres, detecta-se um sinal de alerta que elas estão mais suscetíveis a entrarem para o grupo considerado pobre multidimensional. Todavia, 
vale a pena ressaltar que a maioria dessas áreas encontra-se mais próxima do grupo considerado pobre em termos multidimensionais, mas não foram caracterizadas como tal por não apresentarem o número correspondente ao corte. Com relação às APs consideradas de fato pobres multidimensionalmente, foram observadas apenas seis. Não foram encontradas áreas cujas famílias se encontrem em circunstâncias que caracterizem um estado de pobreza multidimensional grave com pontuações no carpon acima de $50 \%$.

Figura 2 - Mapa do carpon por área de ponderação no ano 2010

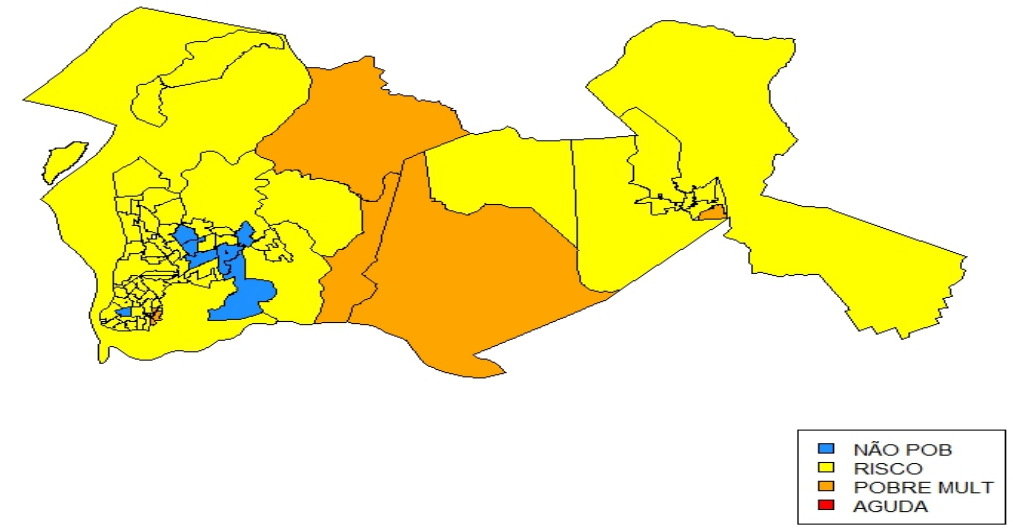

Fonte: Elaborado pelos autores.

A Figura 2 mostra a configuração espacial da pontuação de privação ponderada para toda RMB. Essa configuração exibe três tipos de categorização referentes às carências ponderadas nas áreas de ponderação: as áreas consideradas não pobres que se encontram em Belém e, principalmente, em Ananindeua; as áreas que não são pobres, mas estão vulneráveis à pobreza multidimensional, que representam no total a maioria (60 áreas de ponderação); e as que são classificadas como pobres (6 áreas de ponderação), localizadas, principalmente, no centro do mapa.

\subsection{Padrões de composição dos indicadores dentro das dimensões para o IPM}

O IPM traz em sua composição dimensões que são constituídas por indicadores. Além dos pesos atribuídos a cada dimensão na formação do índice, se torna interessante analisar o peso/importância dos indicadores dentro de cada dimensão, o que propicia uma melhor compreensão do padrão de privação domiciliar dentro de cada AP.

O diagrama de radar apresentado na Figura 3, a seguir, tem um raio para cada um dos indicadores e aponta os pontos fortes de privação em cada um deles. Como exemplo, para o diagrama na Figura 3 foram utilizadas as APs classificadas com melhor e pior posição nos rankings do IPM. De acordo com o radar, na área de ponderação correspondente ao Centro Júlia Seffer, dentro da dimensão educação, o número de indivíduos na família com Ensino Fundamental completo ou incompleto contribuiu relativamente mais do que as crianças dentro da família em idade escolar que não frequentam a escola. Na configuração do padrão de privações na AP 001 no município de Santa Bárbara, a eletricidade se mostrou mais problemática do que questões sanitárias, destino do lixo e a aquisição de bens de 
consumo, visto que apresentou peso maior na dimensão, o que pode eventualmente ter relação com o fato de que o município de Santa Bárbara fica mais afastado da capital (Belém), numa área predominantemente rural.

Figura 3 - Padrões de composição do IPM para duas áreas de ponderação

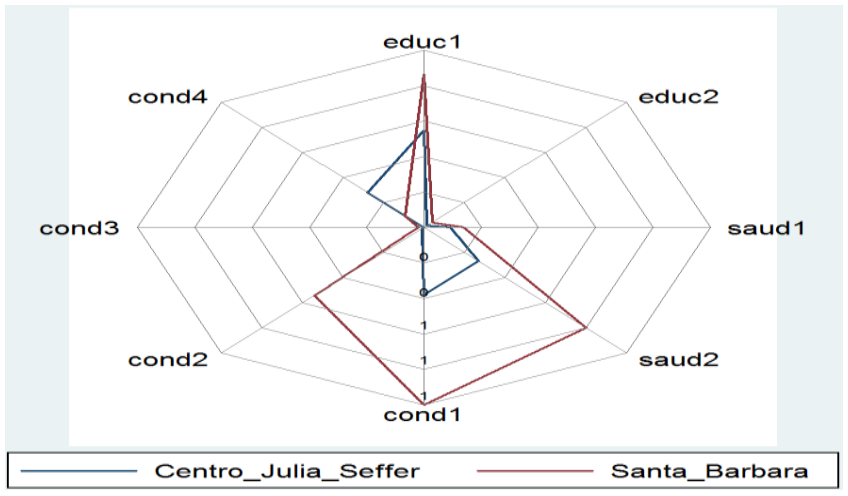

Fonte: Elaboração própria com base nos microdados do Censo 2010.

Como o IPM é formado pelo produto da proporção da população que é considerada pobre (incidência) e da proporção média de privação vivenciada por essas pessoas classificadas como pobres multidimensionais (intensidade), torna-se interessante verificar como esses dois subíndices se relacionam entre si.

A Figura 4, a seguir, plota a intensidade média $(A)$ contra a incidência $(H)$. Nota-se que há uma correlação mesmo que moderada no IPM para a relação $A / H$, isso significa que, para algumas APs, há uma relação análoga entre os dois subíndices: áreas de ponderação com maiores proporções de famílias pobres tendem a ter maior intensidade média de privação.

Figura 4 - Relação entre intensidade $(A)$ e incidência $(H)$ por rendas média e total

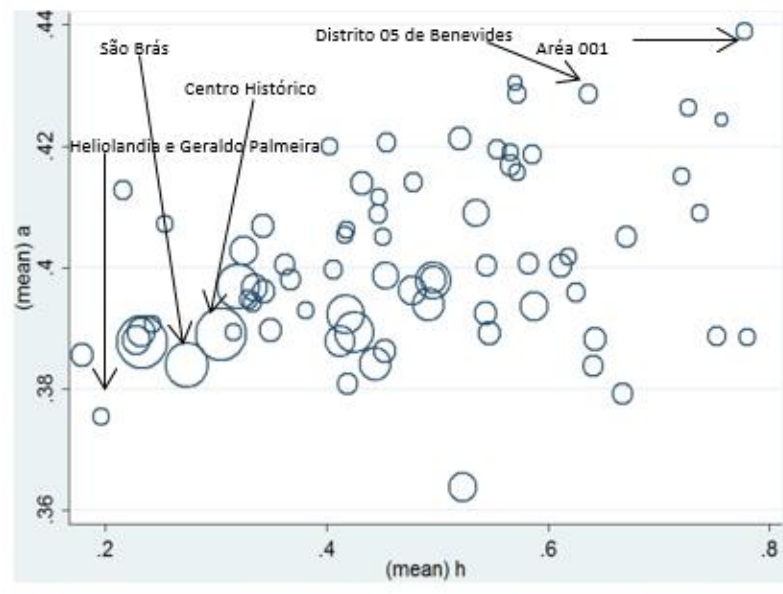

A/ H (Renda Média)

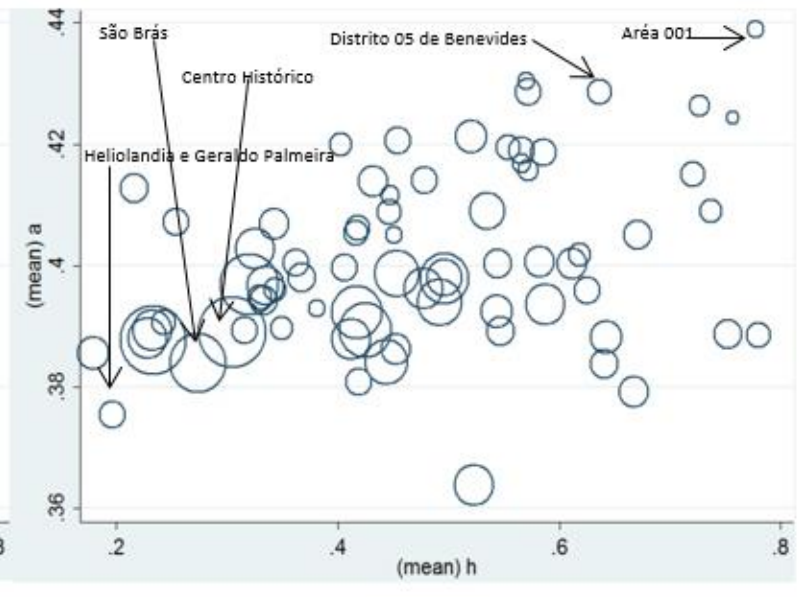

A/ H (Renda Total)

Fonte: Elaboração própria com base nos microdados do Censo 2010.

Outro aspecto importante é verificar como se comportam as rendas média e total nesse contexto: o tamanho da bola na Figura 4 equivale às respectivas rendas. É possível observar que a renda se apresenta como um componente importante na 
condição de pobreza multidimensional das famílias, pois as bolas maiores que significam maiores rendas se localizam do lado esquerdo do gráfico, indicando que as áreas de ponderação com renda per capita e total grandes tendem a possuir menores taxas de proporção de pobres multidimensionais e privação média. O que não se pode deixar de frisar, no entanto, é que há algumas exceções, como é o caso da área de ponderação correspondente a Heliolândia e Geraldo Palmeira, que, apesar de apresentarem renda considerada baixa ou média baixa, possuem IPM baixo se comparado com a média das demais áreas.

Diante de análises sobre pobreza de renda e multidimensional, se torna importante averiguar "até que ponto as mesmas famílias são identificadas como pobres usando duas medidas diferentes" (Alkire e Santos, 2010). Para tal, a partir da identificação de famílias pobres de renda e pobres multidimensionais, foi possível verificar se renda e IPM seriam perfeitamente correlacionados: caso positivo, todas as famílias seriam consideradas pobres (pobres de renda e de IPM) ou não pobres (não pobres de renda e não pobres de IPM); caso contrário, poderiam incorrer os erros tipo I (inclusão) e o tipo II (exclusão), indicando o quão discrepantes são os dois critérios. ${ }^{4}$

Por intermédio dos resultados é possível detectar as probabilidades condicionais à pobreza de renda, ou seja, qual a possibilidade de uma família que é considerada não pobre de renda ser caracterizada como pobre multidimensionalmente e vice-versa. Para a análise da RMB, identificou-se na amostra se cada família que tem renda baixa ou alta é pobre ou não de acordo com o IPM, combinando os domicílios em quatro grupos possíveis (Tabela 4).

Tabela 6 - Referência cruzada das pobrezas de renda e multidimensional (RMB)

\begin{tabular}{lcccc}
\hline & \multicolumn{4}{c}{ POBRE DE RENDA } \\
\cline { 2 - 5 } POBRE & NÃO & NÃO & SIM & TOTAL \\
DE IPM & SIM & 39.42 & 5.64 & $\mathbf{2 9 . 7 3}$ \\
\cline { 2 - 5 } & TOTAL & $\mathbf{6 3 . 5 0}$ & 36.86 & $\mathbf{7 0 . 2 7}$ \\
\hline
\end{tabular}

Fonte: Elaboração própria com base em microdados do Censo 2010

De acordo com o IPM, 24,08\% das famílias foram consideradas não pobres de renda e não pobres de IPM. Os agregados familiares que foram conceituados como pobres de renda, mas não pobres de IPM, abrangem apenas 8.165 famílias (5,64\%). Todavia, vale destacar que houve uma maior ocorrência do erro tipo II em relação ao erro tipo I, pois o percentual de famílias que são consideradas não pobres quando se avalia apenas a pobreza de renda, mas são consideradas multiplamente privadas pelo aspecto multidimensional, foi de 39,42\%, apontando que 87.258 famílias sofrem alguma privação ou uma combinação de privações nas dimensões utilizadas no índice, as quais não seriam captadas se avaliadas apenas pela

\footnotetext{
${ }^{4}$ Conforme Alkire e Santos (2010), o erro tipo I consiste na percentagem de famílias que são pobres de renda, mas não pobres pelo IPM, ao passo que o erro Tipo II tem a ver com a percentagem de famílias que não são pobres de renda, porém pobres em termos multidimensionais.
} 
dimensão da renda. Isso pode estar relacionado ao fato de que o IPM é um índice que busca identificar a pobreza extrema e, portanto, torna-se em certa medida mais adequado para detectá-la do que propriamente mensurações baseadas apenas nos rendimentos.

\section{Considerações finais}

Como já salientado na introdução deste trabalho, apesar do predomínio das abordagens sobre pobreza baseadas em métricas monetárias, a perspectiva multidimensional de pobreza vem ganhando relevância nos últimos anos. Percebese, neste sentido, que essa nova perspectiva tende a captar melhor a complexidade da pobreza, quando avaliada em termos substantivos. O IPM, por exemplo, executa neste sentido uma tipologia de privação que reflete necessidades em serviços básicos e bens essenciais que vão além do que pode ser observado pelos índices de pobreza tradicionais com base em medidas monetárias. O que é uma vantagem para se elaborar perfis descritivos da pobreza em vários de seus aspectos, apesar de muitas vezes a seleção de indicadores ser guiada mais pela disponibilidade de dados do que propriamente pela relevância que o mesmo possa adquirir para a vida das pessoas.

Neste sentido, o IPM, juntamente com medidas monetárias, parecem fornecer informações mais amplas, capazes de promover uma visão mais completa da pobreza e do desenvolvimento de determinada localidade, mesmo quando o mesmo não pode ser utilizado para efeito de comparação ou construção de rankings. Nesse sentido, apesar de suas limitações, este índice, em geral, revela um padrão de pobreza diferente do padrão da pobreza de renda, uma vez que elucida um conjunto diferente de privações sobrepostas, as quais podem ser melhor observadas no agregado familiar.

Com base em análises comparativas entre os resultados da linha de pobreza, do IDHM e do IPM, foi possível neste estudo verificar com maior precisão, as especificidades da pobreza multidimensional estruturalmente existentes na RMB. De uma maneira geral, para o ano de 2010, a pobreza de renda e pobreza multidimensional apresentam desempenhos bastante diferentes, no qual algumas áreas consideradas menos pobres em termos multidimensionais, não os são, necessariamente, as mesmas menos pobres quando avaliadas em termos de renda. Além disso, observando as proporções de pobres, percebe-se que nas áreas de ponderação em que praticamente coincidem os indicadores de renda e o multidimensional, as proporções não estão na mesma posição na classificação geral dos indicadores sintéticos de avaliação sobre pobreza.

Os resultados também propiciaram a constatação no que tange à composição de privações domiciliares entre as áreas de ponderação que, de maneira geral, as famílias residentes na $\mathrm{RMB}$ são afetadas por diferentes tipos de privações em conjunto. Os dados mostraram que a incidência da pobreza multidimensional em 2010 ainda é bastante elevada, mesmo após inúmeros avanços sociais vastamente relatados na literatura nos últimos 15 anos. Nota-se que a preponderância das privações com relação à escolaridade e à saúde, por sua vez, não torna menos importante a contribuição significativa da dimensão "Condições de vida". Um dos aspectos interessantes nesse sentido, é a consideração da importância da dimensão 
"Educação", o que reforça, de certa maneira, os resultados relatados inicialmente na avaliação agregada, feita com base apenas no IDHM para os municípios da RMB.

Traçadas a caracterização da pobreza multidimensional (por intermédio do IPM) e as análises da proporção de pobres de renda, foi possível inferir que a pobreza é maior e mais complexa na perspectiva multidimensional do que aparece no âmbito da renda. Por isso, torna-se vital compreender a composição das privações entre as diferentes APs, de maneira que as intervenções públicas possam trabalhar para resolver de forma mais específica e eficaz as carências das famílias por serviços básicos. A magnitude e a concentração da pobreza analisada, levando em consideração a utilização de um índice composto por várias dimensões, se mostraram mais complexas e abrangentes na identificação das famílias e no acúmulo de privações que elas experimentam. Prova disso é que, de uma forma geral, ao se comparar a proporção de pobres multidimensionais com a proporção de pobres de renda (com base nos dados do IETS para 2011) (Rocha, 2011), mesmo com a defasagem de um ano, verificou-se que enquanto em 2011 a proporção de pobres de renda na RMB era de $23 \%$, a incidência da pobreza multidimensional (conforme dados do censo) era de $46 \%$ em 2010.

Percebe-se que os resultados se mostraram satisfatórios na medida em que fornecem uma visão mais ampla da pobreza multidimensional, assim como quais áreas dentro de cada município apresentam maior incidência e intensidade da pobreza. Porém, faz-se necessário em um próximo estudo a focalização da análise por subgrupos populacionais, para identificar grupos/indivíduos cujas privações sobrepostas sejam maiores e estejam mais evidentes, para assim poder captar diferenças entre gênero, idade, raça, dentre outras especificidades. Da mesma forma, possibilitando o direcionamento das ações governamentais não apenas para a AP, mas para grupos/indivíduos considerados mais vulneráveis na população.

Acredita-se que um indicador multidimensional mais robusto deveria levar em consideração a realidade do cotidiano das pessoas consideradas pobres: como o acesso ao crédito, à exclusão social, à segurança alimentar, à desigualdade de gênero e raça, a terra e as limitações em relação à condição de agente (capacitação) das pessoas, dentre outras características ou dimensões da pobreza que fazem parte do dia a dia das populações que vivem em regiões mais carentes e que não podem ser negligenciadas como espaços de avaliação dos níveis de pobreza vistos por um índice multidimensional o mais amplo possível.

No entanto, mesmo reconhecendo as limitações do IPM, pode-se considerar que estudos que avancem, mesmo que limitadamente na incorporação de diferentes dimensões da pobreza, são fundamentais para que se possa avaliar, mesmo que preliminarmente, com maior detalhe a natureza multidimensional da pobreza e suas implicações sob o ponto de vista do estudo sobre a qualidade de vida nas grandes cidades brasileiras. Isso porque o IPM abrange dimensões consideradas fundamentais e que, de certo modo, caracterizam a condição de pobreza dos indivíduos, condições que vão muito além da pobreza avaliada em seus aspectos apenas relacionados à insuficiência de renda.

\section{REFERÊNCIAS}


ALKIRE, SABINA, AND MARIA EMMA SANTOS. Acute Multidimensional Poverty: A New Index for Developing Countries. OPHI Working Papers 38 (2010). University of Oxford.

ALKIRE, SABINA. Dimensions of Human Development. World Development 30, n. 2 (2002): 181-205.

ALKIRE, SABINA; FOSTER, JAMES. Counting and multidimensional poverty measurement. Journal of Public Economics, v. 95, p. 476-487, 2011.

ALKIRE, SABINA; SETH, SUMAN. Multidimensional poverty reduction in India between 1999 and 2006: Where and how?. World Development, v. 72, p. 93-108, 2015 .

ANGULO, ROBERTO; DÍAZ, YADIRA; PARDO, RENATA. The Colombian multidimensional poverty index: Measuring poverty in a public policy context. Social Indicators Research, v. 127, n. 1, p. 1-38, 2016.

ATKINSON, ANTHONY BARNES. Multidimensional Deprivation: Contrasting Social Welfare and Counting Approaches. Journal of Economic Inequality 1, n. 1 (2003): 5165.

ATLAS DO DESENVOLVIMENTO HUMANO NO BRASIL. IDHM Municipal 2000 e 2010. 2013. http://www.atlasbrasil.org.br/2013/pt/consulta/.

BARROS, RICARDO PAES, CARVALHO, MIRELA DE, FRANCO, SAMUEL. O Índice de Desenvolvimento da Família (IDF). Rio de Janeiro: Ipea, 2003. (Texto para Discussão, n. 986).

CALLANDER, EMILY J.; SCHOFIELD, DEBORAH J.; SHRESTHA, RUPENDRA N. Capacity for freedom-a new way of measuring poverty amongst Australian children. Child Indicators Research, v. 5, n. 1, p. 179-198, 2012.

CARVALHO, MÁRCIA MARQUES DE, KERSTENETZKY, CELIA LESSA E DEL VECCHIO, RENATA. Uma aplicação da teoria dos conjuntos fuzzy na análise da pobreza: 0 caso das regiões metropolitanas do sudeste brasileiro - 2000. Anais do XXXV Encontro Nacional de Economia, ANPEC, Recife, 2007.

CHZHEN, YEKATERINA; FERRONE, LUCIA. Multidimensional child deprivation and poverty measurement: Case study of Bosnia and Herzegovina. Social Indicators Research, v. 131, n. 3, p. 999-1014, 2017.

COMIM, FLÁVIO V., E BAGOLIN, IZETE PENGO. Aspectos qualitativos da pobreza no Rio Grande do Sul. Ensaios FEE 23 (2002): 467-90.

DEÁK, CSABA, AND SUELI R. SCHIFFER. O processo de urbanização no Brasil. São Paulo: EDUSP, 2010. 
DINIZ, MARCELO BENTES, DINIZ, MARCOS MONTEIRO. Um indicador comparativo de pobreza multidimensional a partir dos Objetivos do Desenvolvimento do Milênio. Econômica Aplicada 13, n. 3 (2009): 399-423. http://dx.doi.org/10.1590/S141380502009000300003 .

DUCLOS, JEAN-YVES, SAHA, DAVID E. YOUNGER, STEPHEN D.. Robust Multidimensional Poverty Comparisons. The Economic Journal 116 (2006): 943-68.

FONSECA, OSWALDO. Aplicação de métodos de análise espacial e da teoria dos conjuntos nebulosos em estudos sobre pobreza. PhD diss., Universidade Federal do Rio de Janeiro, 2003.

INSTITUTO BRASILEIRO DE GEOGRAFIA E ESTATÍSTICA - IBGE. Censo Demográfico 2000 e 2010. http://www.ibge.gov.br.

JANNUZZI, P.M.; SOUSA, M.F. Contribuição para a agenda de objetivos de desenvolvimento sustentável: Indicador de Pobreza Multidimensional de Pobreza para América Latina inspirada em proposta preliminar da Cepal. Estudo Técnico SAGI, n. 13, 2016. Disponível em: https://aplicacoes.mds.gov.br/sagi/portal/. Acesso em: 14 maio, 2019.

KAGEYAMA, ANGELA, AND RODOLFO HOFFMANN. Pobreza no Brasil: uma perspectiva multidimensional. Economia e Sociedade 15, n. 1 (2006): 79-112.

KAKWANI, NANAK, AND JACQUES SILBER, ed. Quantitative Approaches to Multidimensional Poverty Measurement. Palgrave Macmillan, Basingstoke, 2008b.

KAKWANI, NANAK, AND JACQUES SILBER, ed. The Many Dimensions of Poverty. Palgrave MacMillan, Basingstoke, 2008a.

KHAN, ATTA ULLAH ET AL. Poverty assessment as a multidimensional socioeconomic concept: the case of the Rawalpindi region in Pakistan. Asia Pacific Journal of Social Work and Development, v. 24, n. 4, p. 238-250, 2014.

LEVINE, SEBASTIAN; MUWONGE, JAMES; BATANA, YELE MAWEKI. A robust multidimensional poverty profile for Uganda. Journal of human development and capabilities, v. 15, n. 4, p. 369-390, 2014.

LOPES, HELGER MARRA, MACEDO, PAULO BRIGIDO ROCHA, MACHADO, ANA FLAVIA. Análise de pobreza com indicadores multidimensionais: uma aplicação para Brasil e Minas Gerais. Revista de Economia Contemporânea 9, n. 1 (2005): 125-52.

MONTOYA, ÁLVARO JOSÉ ALTAMIRANO; TEIXEIRA, KARLA MARIA DAMIANO. Multidimensional poverty in Nicaragua: Are female-headed households better off?. Social Indicators Research, v. 132, n. 3, p. 1037-1063, 2017.

MUSHONGERA, DARLINGTON; ZIKHALI, PRECIOUS; NGWENYA, PHINDILE. A multidimensional poverty index for Gauteng province, South Africa: evidence from Quality of Life Survey data. Social Indicators Research, v. 130, n. 1, p. 277-303, 2017. 
NEDER, HENRIQUE DANTAS. Um estudo sobre a pobreza rural com abordagem multivariada. Congresso da Sociedade Brasileira de Economia e Sociologia Rural (Sober), Rio Branco, 2008. http://www.sober.org.br/palestra/9/633.pdf.

PICOLLOTO, VOLNEI, COMIM, FLÁVIO V., BAGOLIN, IZETE PENGO, PORTO JUNIOR, SABINO DA SILVA, E ÁVILA, RODRIGO. Avaliação multidimensional da pobreza um exercício piloto para Porto Alegre e região metropolitana. Encontro de Economia da Região Sul, Curitiba, 2007.

PINILLA-RONCANCIO, MÓNICA; SILVA, RAQUEL. Children in Angola: Poverty, deprivation and child labour. Child Indicators Research, v. 11, n. 3, p. 981-1005, 2018.

PROGRAMA DAS NAÇÕES UNIDAS PARA O DESENVOLVIMENTO - PNUD. Relatório de Desenvolvimento Humano de 2013. Canadá, 2013.

QI, DI; WU, YICHAO. A multidimensional child poverty index in China. Children and Youth Services Review, v. 57, p. 159-170, 2015.

ROCHA, SÔNIA. Linhas de pobreza 1985-2009. 2011. http:// www.iets.org.br/ article.php3?id_article=915.

SABOOR, ABDUL ET AL. Multidimensional deprivations in Pakistan: Regional variations and temporal shifts. The Quarterly Review of Economics and Finance, v. 56, p. 57-67, 2015.

SEN, AMARTYA K. Poverty: an ordinary approach to measurement. Econometrica 44, n. 2 (1976): 219-31. DOI: 10.2307/1912718.

SEN, AMARTYA K. CHOICE, Welfare and Measurement. Cambridge: Havard University Press, 1982.

SEN, AMARTYA K. Commodities and Capability. Oxford: Oxford University Press, 1985.

SEN, AMARTYA K. Desigualdade reexaminada. Rio de Janeiro: Record, 2001.

SEN, AMARTYA K. Development as Freedom. New York: Knopf, 1999a.

SEN, AMARTYA K. Sobre ética e economia. São Paulo: Companhia das Letras, 1999b.

SEN, AMARTYA K., and James Foster. On Economic Inequality. Oxford: Claredon Press, 1997.

SERRA, A.S. Pobreza multidimensional no Brasil rural e urbano. Tese (doutorado) Universidade Estadual de Campinas, Instituto de Economia. Campinas, SP, 2017.

SOLAYMANI, SAEED; KARI, Fatimah. Poverty evaluation in the Malaysian fishery community. Ocean \& Coastal Management, v. 95, p. 165-175, 2014. 
VAZ, A.C.N.; JANNUZZI, P.M. Indicador de Pobreza Multidimensional como síntese dos efeitos da abordagem multissetorial do Plano Brasil Sem Miséria. Revista

Brasileira de Monitoramento e Avaliação, Brasília, DF, n. 8, 2014.

Danuzia Lima Rodrigues. Economista, Professora do departamento de Economia da UNIFESSPA. E-mail dannylrc2005@hotmail.com

Ricardo Bruno Nascimento dos Santos. Doutor em Economia, Professore e Pesquisador da UFPA. E-mail ricardobns@gmail.com

Izete Pengo Bagolin. Economista Graduada pela UFSM, Mestre em Economia Rural pela UFRGS, Doutora em Economia pela UFRGS. Professora do PPGE/PUCRS. E-mail izete.bagolin@pucrs.br

Danilo Araujo Fernandes. Professor e Pesquisador da UFPA. E-mail danfernandes2@hotmail.com

Como citar: RODRIGUES, Danuzia Lima et al. O Programa Bolsa Família Estimula a Permanência dos Trabalhadores Brasileiros de Baixa Renda no Mercado Informal?. Redes (St. Cruz Sul, Online), Santa Cruz do Sul, v. 25, p. 2251-2273, 2020. ISSN 1982-6745. doi:https://doi.org/10.17058/redes.v25i0.14166.

\section{CONTRIBUIÇÃO DE CADA AUTOR}

Conceituação (Conceptualization): Danuzia, Izete, Ricardo e Danilo Curadoria de Dados (Data curation): IBGE

Análise Formal (Formal analysis): Danuzia, Ricardo, Izete e Danilo

Obtenção de Financiamento (Funding acquisition):

Investigação/Pesquisa (Investigation): Danuzia, Ricardo

Metodologia (Methodology): Danuzia, Ricardo

Administração do Projeto (Project administration): Ricardo e Danilo

Recursos (Resources): CNPq, Conselho Nacional de Desenvolvimento Científico e

Tecnológico - Brasil (n³06707/2017-9) e bolsa CNPQ GM.

Software: R e Stata

Supervisão/orientação (Supervision): Ricardo

Validação (Validation): Ricardo, Izete e Danilo

Escrita - Primeira Redação (Writing - original draft): Danuzia

Escrita - Revisão e Edição (Writing - review \& editing): Izete, Danilo, Danuzia e Ricardo

Fontes de financiamento: O presente trabalho foi realizado com apoio do CNPq, Conselho Nacional de Desenvolvimento Científico e Tecnológico - Brasil ( $\left.n^{\circ} 306707 / 2017-9\right)$ e Bolsa de Doutorado CNPQ GM. 